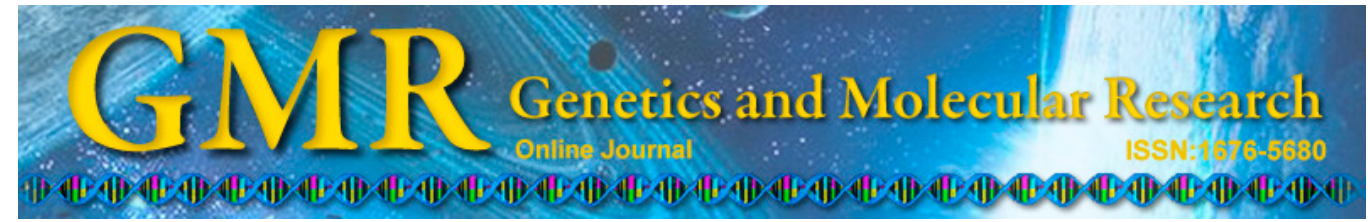

\title{
Analysis of biochemical compounds and differentially expressed genes of the anthocyanin biosynthetic pathway in variegated peach flowers
}

\author{
D. Hassani, H.L. Liu, Y.N. Chen, Z.B. Wan, Q. Zhuge and S.X. Li \\ Co-Innovation Center for Sustainable Forestry in Southern China, \\ College of Forestry, Nanjing Forestry University, Nanjing, China \\ Corresponding author: S.X. Li \\ E-mail: shuxianli@njfu.com.cn
}

Genet. Mol. Res. 14 (4): 13425-13436 (2015)

Received June 8, 2015

Accepted September 17, 2015

Published October 28, 2015

DOI http://dx.doi.org/10.4238/2015.October.28.4

\begin{abstract}
Variegated plants are highly valuable in the floricultural market, yet the genetic mechanism underlying this attractive phenomenon has not been completely elucidated. In this study, we identified and measured different compounds in pink and white flower petals of peach (Prunus persica) by high-performance liquid chromatography and liquid chromatography/mass spectrometry analyses. No cyanidin-based or pelargonidin-based compounds were detected in white petals, but high levels of these compounds were found in pink petals. Additionally, we sequenced and analyzed the expression of six key structural genes in the anthocyanin biosynthesis pathway (CHI, CHS, DFR, F3'H, ANS, and UFGT) in both white and pink petals. Quantitative real-time polymerase chain reaction revealed all six genes to be expressed at greatly reduced levels in white flower petals, relative to pink. No allelic variations were found in the transcribed sequences. However, alignment of transcribed and genomic sequences of the $A N S$ gene detected alternative splicing, resulting in transcripts
\end{abstract}


of 1.071 and $942 \mathrm{bp}$. Only the longer transcript was observed in white flower petals. Since ANS is the key intermediate enzyme catalyzing the colorless leucopelargonidin and leucocyanidin to substrates required for completion of anthocyanin biosynthesis, the $A N S$ gene is implicated in flower color variegation and should be explored in future studies. This article, together with a previous transcriptome study, elucidates the mechanism underlying peach flower color variegation in terms of the key structural genes involved in anthocyanin biosynthesis.

Key words: Peach; Flower color variegation; HPLC; LC/MS; Anthocyanin biosynthesis; Structural gene

\section{INTRODUCTION}

Flower color variegation of plants is a highly valuable feature in the floricultural market. Molecular mechanisms of flower variegation have been investigated in various plant species (Liu et al., 2001; Iida et al., 2004). Studies reveal that some of this variegation is caused by the insertion of transposable elements into structural genes associated with the anthocyanin biosynthetic pathway (Inagaki et al., 1994; Quattrocchio et al., 1999). Chimeric flowers are frequently observed on peach trees (Figure S1) and variegation in anthocyanin production in both vegetative and reproductive tissues has been studied in the peach cultivar "Pillar". Results show that this phenotype is heritable, although the degree of variegation differs according to the genetic background of outcross progeny (Chaparro et al., 1995). To date, the genetic mechanisms responsible for this unstable phenotype in peach have not been elucidated. Because of the complexity of the flavonoid biosynthetic pathway, the exact genetic mechanisms underlying the generation of flower pigmentation chimeras may differ among species (Chaparro et al., 1995). Thus, studying the genetic mechanism underlying peach flower variegation will improve our understanding and may lead to novel discoveries about this attractive phenomenon. In the past decades, accumulated knowledge of the biochemistry and genetic control of flower pigmentation, together with the incredible genomic resources developed in peach (Verde et al., 2013; Chen et al., 2014), have enabled us to dissect the molecular basis for color variegation in peach flowers.

Flower pigmentation involves a multi-enzymatic biosynthetic pathway, in which several enzymatic reactions result in the production and accumulation of flavonoid compounds. Flavonoids also play important roles in many other biological processes, such as UV protection (Li et al., 1993), pathogen defense (Treutter, 2005), and pollen viability (Taylor and Jorgensen, 1992). There are three major classes of plant flavonoids (anthocyanins, proanthocyanins, and flavonols), which are synthesized via the branched flavonoid biosynthetic pathway. Anthocyanins, the most important flavonoid class, are the main pigments in flowers and fruits; these pigments are vital for animal and insect attraction, which are important for pollination and seed dispersal (Winkel-Shirley, 2001). The basic anthocyanins consist mainly of pelargonidin, cyanidin, and delphinine; various modifications of these compounds, such as glycosylation, acylation, and methylation, contribute to diversity in flower color (Morata et al., 2007). Intensive studies have been conducted in flower pigmentation, especially in snapdragon (Antirrhinum majus) and petunia (Petunia hybrida) (Quattrocchio et al., 1993). More recently, petunia has become the organism of choice for studies of flavonoid biosynthetic genes. Although the anthocyanin biosynthetic pathways in most plants share common reactions, different types of 
anthocyanins are produced in different plant species (Holton and Cornish, 1995). For instance, petunia does not normally synthesize pelargonidin, whereas snapdragon is incapable of producing delphinidin (Holton and Cornish, 1995). Peach is found to produce large amounts of cyanidin-3-O-gycoside (Jaganath and Crozier, 2010).

In this study, we investigated the compounds produced in flower petals of different colors (white and pink) from a marked, variegated peach tree. In addition, we also sequenced and analyzed key structural genes involved in anthocyanin biosynthesis that were previously found to be differentially expressed between pink and white peach flower petals (Chen et al., 2014). The expression levels of these genes were subsequently measured using quantitative real-time polymerase chain reaction (qRT-PCR). Taken together, the results of the study provide new insights into the genetic mechanisms controlling the color variegation of peach flowers.

\section{MATERIAL AND METHODS}

\section{Plant materials}

White and pink petals were collected from a marked tree of Prunus persica f. versicolor, bearing variegated flowers, in Nanjing Lovers' Garden, Jiangsu, China, in April 2013. Petals were immediately frozen in liquid nitrogen and stored at $-80^{\circ} \mathrm{C}$ until RNA extraction. The field studies did not involve any endangered or protected species, and the administrative office of Nanjing Lovers' Garden authorized sample collection.

\section{Sample preparation for high-performance liquid chromatography (HPLC) analysis}

For white and pink flowers, approximately $50-100 \mathrm{mg}$ petal material was ground separately in clean autoclaved mortars. Then, samples were extracted with $6 \mathrm{~mL}$ acidic methanol solution $\left(\mathrm{CH}_{3} \mathrm{OH}: \mathrm{HCl}: \mathrm{H}_{2} \mathrm{O}, 70: 0.1: 29.9\right)$, and shaken using a vortex mixer at $4{ }^{\circ} \mathrm{C}$ for $24 \mathrm{~h}$, following the protocol described by Jia et al. (2008). The extractions of white and pink petals were each filtered through a $0.22-\mu \mathrm{m}$ membrane (Membrana, Germany), and the filtrates were used for flavonoid detection and identification.

\section{Identification of flavonoids by HPLC analysis}

Chromatographic analysis was performed on an HPLC machine equipped with an E2695 HPLC pump, an XBridge C18 column ( $5 \mu \mathrm{m}, 4.6$ x $250 \mathrm{~mm})$, and a Dionex Photodiode Array DAD-2998 Detector (Waters, USA). The column temperature was set at $30^{\circ} \mathrm{C}$ and 50 $\mu \mathrm{L}$ filtrates of white or pink flower petals were injected. The flow rate was $0.8 \mathrm{~mL} / \mathrm{min}$ and the mobile phase consisted of acetonitrile $\left(\mathrm{CH}_{3} \mathrm{CN}\right)$ as solvent $\mathrm{A}$ and $0.05 \% \mathrm{H}_{3} \mathrm{PO}_{4}-\mathrm{H}_{2} \mathrm{O}$ as solvent B. The linear gradient profile of solvent B was $10 \%$ at $0 \mathrm{~min}, 20 \%$ at $30 \mathrm{~min}, 30 \%$ at $50 \mathrm{~min}$, $40 \%$ at $60 \mathrm{~min}, 50 \%$ at $80 \mathrm{~min}$, and then a return to $10 \%$ at $90 \mathrm{~min}$. The first detection was made in the photodiode apparatus, set to $515 \mathrm{~nm}$ as the preferred wavelength.

\section{Identification of flavonoids by liquid chromatography-mass spectometry (LC-MS)}

Samples were injected into a Thermo LTQ Orbitrap XL HPLC system (Thermo Fisher Scientific, USA) equipped with an LC/MSD Trap VL electrospray ion (ESI) MS for analysis 
and confirmation of the identified flavonoids. The MS analysis was performed in positive ionization mode, monitoring the protonated molecular ions. Heater temperature was set at $200^{\circ} \mathrm{C}$, sheath gas flow rate at $25 \mathrm{AU}$, aux gas flow rate at $5 \mathrm{AU}$, spray voltage at $3.50 \mathrm{kv}$, capillary temperature at $350^{\circ} \mathrm{C}$, capillary voltage at $-35.00 \mathrm{v}$, and tube lens at $-118.42 \mathrm{v}$. Scan range of $\mathrm{m} / \mathrm{z}$ was set to 100-800 mass units. In this experiment, solvent A was $90 \%$ methanol and solvent $\mathrm{B}$ was autoclaved $\mathrm{H}_{2} \mathrm{O}$. The flow rate was set at $0.2 \mathrm{~mL} / \mathrm{min}$.

Data was recorded with the Chromeleon software (Dionex, USA). The pink and white petal extracts were checked for glycosylated derivatives of cyanidin, pelargonidin, and peonidin, according to mass to charge ratio $(\mathrm{m} / \mathrm{z})$ and molecular weight. Derivatives included pelargonidin-3-glucoside, pelargonidin-3-(6"-ethylmalonyl glucoside), cyaniding-3-glucoside, cyanidin-3-(6"-malonyl glucoside), cyanidin-3-(6"-ethylmalonyl glucoside), peonidin-3-glucoside, and peonidin-3-(6"-ethylmalonyl glucoside).

\section{Total RNA isolation and cDNA synthesis}

Total RNA was separately extracted from petals of pink and white flowers using RNeasy Plant Mini Kit (Qiagen, Germany), according to manufacturer protocol. The integrity and quality of the isolated RNA were assessed using agarose gel electrophoresis. RNA concentration was measured using a Nanodrop-2000 spectrophotometer (Thermo Fisher Scientific, USA). Prior to reverse transcription, total RNA was treated with DNase (DNase I Kit, Takara, Japan). Then, cDNA was transcribed using a reverse transcription kit (Promega, USA). Finally, the synthesized cDNA was quantified with a spectrophotometer (Eppendorf, Germany) set to a wavelength of $260 \mathrm{~nm}$.

\section{Isolating the full-length cDNA of flavonoid biosynthetic genes}

We first amplified part of each gene. Based on the retrieved flavonoid biosynthetic genes of $P$. persica that were available in GenBank, gene-specific primers were designed (Table S1), including $C H S, C H I, F 3^{\prime} H, A N S, D F R$, and UFGT. The first round of PCR was performed using an LA Taq ${ }^{\mathrm{R}} \mathrm{Kit}$ (Takara). PCR was carried out by denaturing the cDNA at $94^{\circ} \mathrm{C}$ for $3 \mathrm{~min}$; followed by 30 cycles of $30 \mathrm{~s}$ at $94^{\circ} \mathrm{C}, 30 \mathrm{~s}$ at $55^{\circ}-59^{\circ} \mathrm{C}$ (depending on the primer $\mathrm{T}_{\mathrm{m}}$ ), and $60 \mathrm{~s}$ at $72^{\circ} \mathrm{C}$; and a final extension of $300 \mathrm{~s}$ at $72^{\circ} \mathrm{C}$.

The 3'-RACE and 5'-RACE were conducted with a SMARTer RACE cDNA amplification kit (Clontech Laboratories, USA), following the manufacturer protocol. Gene-specific primers were designed according to the 3'- and 5'-sequences of the retrieved reference genes (Table S2). The 3'-RACE PCR was carried out by denaturing the cDNA at $94^{\circ} \mathrm{C}$ for $3 \mathrm{~min}$; followed by 20 cycles of $30 \mathrm{~s}$ at $94^{\circ} \mathrm{C}, 30 \mathrm{~s}$ at $68^{\circ} \mathrm{C}$, and $3 \mathrm{~min}$ at $72^{\circ} \mathrm{C}$. The $5^{\prime}-\mathrm{RACE}$ PCR used the following cycle profile: $3 \mathrm{~min}$ at $94^{\circ} \mathrm{C}$; followed by 5 cycles of $30 \mathrm{~s}$ at $94^{\circ} \mathrm{C}$ and $3 \mathrm{~min}$ at $72^{\circ} \mathrm{C}$; 5 cycles of $30 \mathrm{~s}$ at $94^{\circ} \mathrm{C}, 30 \mathrm{~s}$ at $70^{\circ} \mathrm{C}$, and $3 \mathrm{~min}$ at $72^{\circ} \mathrm{C}$; and finally, 20 cycles of $30 \mathrm{~s}$ at $94^{\circ} \mathrm{C}, 30 \mathrm{~s}$ at $68^{\circ} \mathrm{C}$, and $3 \mathrm{~min}$ at $72^{\circ} \mathrm{C}$.

Sequence assembly was performed using DNAMAN (v6.0.40, Lynnon Biosoft, USA). To verify gene assembly, specific primers were designed to amplify the full lengths of corresponding genes (Table S3 $)$. PCR was carried out by denaturing the cDNA at $94^{\circ} \mathrm{C}$ for 3 min; followed by 30 cycles of $30 \mathrm{~s}$ at $94^{\circ} \mathrm{C}, 30 \mathrm{~s}$ at $55^{\circ}-59^{\circ} \mathrm{C}$ (depending on the primer $\mathrm{T}_{\mathrm{m}}$ ), and $60 \mathrm{~s}$ at $72^{\circ} \mathrm{C}$; and a final extension of $300 \mathrm{~s}$ at $72^{\circ} \mathrm{C}$. Genomic DNA was isolated from pink 
and white flower petals using the CTAB method. The ANS gene, along with 5-kb upstream and downstream flanking sequences, were amplified from the genomic DNA template.

\section{Purifying, cloning, and sequencing}

The PCR product from each round was run on a $1 \%$ agarose gel, from which the desired band was extracted and purified using the AxyPrep DNA Gel Extraction Kit (Axygen Biosciences, USA). Extract concentrations were quantified with a Nanodrop-2000 spectrophotometer (Thermo Fisher Scientific).

The extracts of PCR products were transformed into the pGEM-T Easy vector (Promega) and cloned into JM 109 high-efficiency competent cells. The transformed cells were then cultured on LB medium containing Ampicillin $(50 \mu \mathrm{g} / \mathrm{mL})$. Colony PCR was carried out with specific primers in order to confirm the success of transformations from each round of PCR. For each gene, three colonies representing successful transformations were selected for sequencing by the Jinsirui Company of Nanjing, China (http://www.genscript.com.cn).

Sequence alignment was carried out with the BioXM software (v2.6) (http:// www.home.njau.edu.cn/bioxm). The sequences obtained from pink and white petals were BLASTed against the NCBI nucleotide and protein database (http://blast.ncbi.nlm.nih.gov/ Blast.cgi). Pairwise alignment was performed using DNAMAN (v6.0.40, Lynnon Biosoft). Protein structure homology was examined using the online SWISS-MODEL toolkit (http:// swissmodel.expasy.org) (Guex and Peitsch, 1997; Schwede et al., 2003; Arnold et al., 2006). A conserved domain search and a self-optimized prediction from multiple-alignment were performed using CD-Search (Marchler and Bryant, 2004) and ANTHEPROT 2.0 (Geourjon and Deleage, 1995), respectively.

\section{Quantitative real-time PCR analysis}

The expression levels of the genes isolated from pink and white petals were examined by qRT-PCR using the 7500 Real-Time PCR System (Applied Biosystems, USA) with Fast Start Universal SYBR Green Master Mix (Roche Diagnostics, Germany). Three sets of gene-specific primers were designed for each gene (Table S4) using the GenScript Real-Time PCR (TaqMan) online primer design tool (https://www.genscript.com/ssl-bin/app/primer). With reference to relevant studies (Gutha et al., 2010), several genes were selected and tested as internal controls (Table S5). The qRT-PCR was performed in a $20-\mu \mathrm{L}$ reaction volume, as described previously in Chen et al. (2014). The best gene-specific primers and internal control for qRT-PCR were selected based on their electrophoresis profiles. qRT-PCR was performed in three independent experimental replicates. In each, triplicate samples of white and pink petals were used. Calculation of relative expression level was performed using the $2^{-\Delta \Delta \mathrm{Ct}}$ method (Livak and Schmittgen, 2001).

\section{RESULTS}

\section{HPLC and LC-MS analysis}

Figure 1 shows the HPLC anthocyanin profiles of white and pink petals of peach flowers. By comparison of their HPLC retention times with the appropriate standards and 
those of anthocyanins obtained from published data (De Pascual-Teresa et al., 2002), seven glycosylated derivatives of cyanidin, pelargonidin, and peonidin were detected in pink petal extracts (Figure 1A). However, none of these compounds were detected in white extracts, at the same wavelength (Figure 1B). The identities of the detected compounds were further confirmed by LC-MS analysis, which showed positively mono-charged molecular ions at $\mathrm{m} / \mathrm{z}$ ratios of $449,433,463,535,563,547$, and 577 for the peaks produced by pink petal extracts (Table 1). In contrast, no signal was detected for extracts from white flowers.

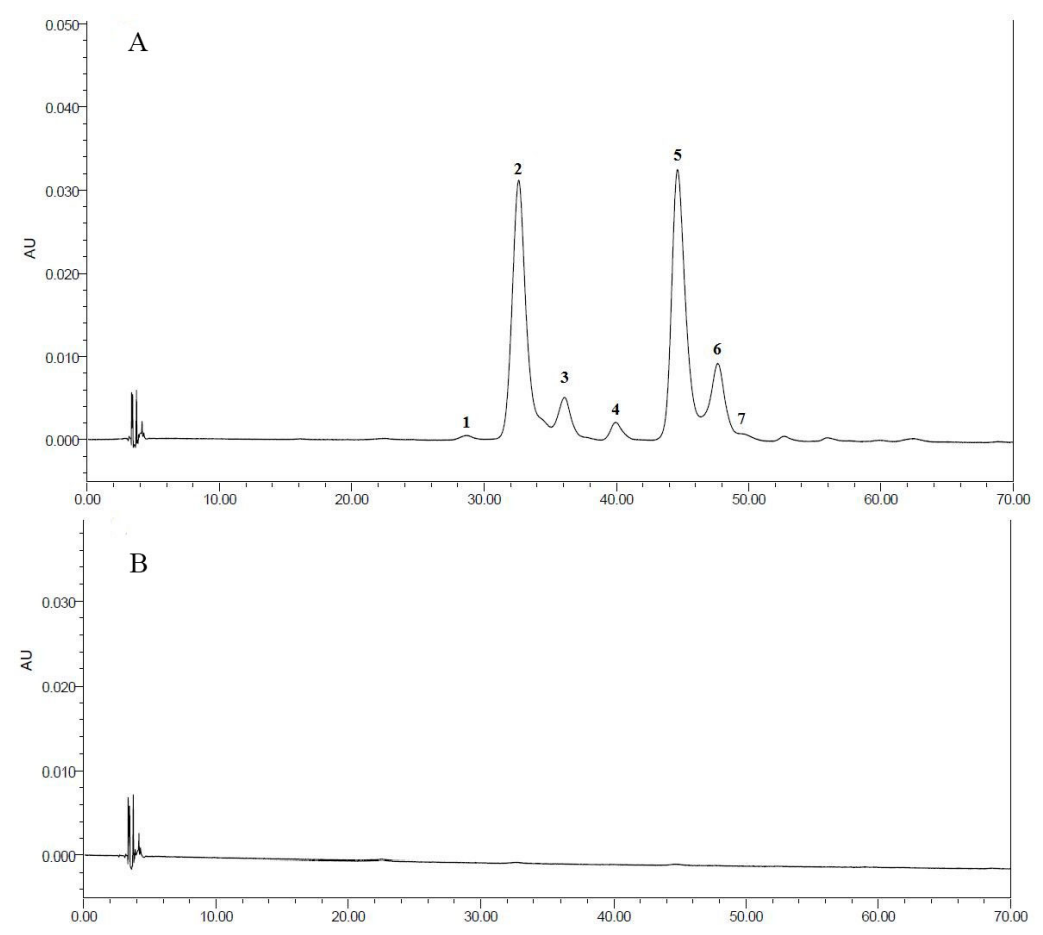

Figure 1. HPLC chromatogram at $515 \mathrm{~nm}$ for pink (A) and white (B) flower petal extracts. The information of peaks 1-7 was described in Table 1.

Table 1. Chromatographic and spectral characteristics of peaks in Figure 1.

\begin{tabular}{|c|c|c|c|c|c|c|}
\hline \multirow[t]{2}{*}{ Peak } & \multirow[t]{2}{*}{ Compound } & \multirow[t]{2}{*}{ RT (min) } & \multirow[t]{2}{*}{$\lambda_{\max }(\mathrm{nm})$} & \multirow[t]{2}{*}[\mathrm{m}/\mathrm{z}]{$^{+}$} & \multicolumn{2}{|c|}{ Detection } \\
\hline & & & & & White & Pink \\
\hline 1 & Cyanidin-3-glucoside & 28.0 & 515 & 449 & ND & $\mathrm{D}$ \\
\hline 2 & Pelargonidin-3-glucoside & 34.0 & 515 & 433 & ND & $\mathrm{D}$ \\
\hline 3 & Peonidin-3-glucoside & 37.0 & 515 & 463 & ND & $\mathrm{D}$ \\
\hline 4 & Cyanidin-3-(6"-malonyl glucoside) & 39.8 & 515 & 535 & ND & $\mathrm{D}$ \\
\hline 5 & Cyanidin-3-(6"-ethylmalonyl glucoside) & 46.7 & 515 & 563 & ND & $\mathrm{D}$ \\
\hline 6 & Pelargonidin-3-(6"-ethylmalonyl glucoside) & 48.9 & 515 & 547 & ND & $\mathrm{D}$ \\
\hline 7 & Peonidin-3-(6"-ethylmalonyl glucoside) & 49.9 & 515 & 577 & ND & D \\
\hline
\end{tabular}

$\mathrm{RT}=$ retention time; $\lambda_{\max }=$ wavelength at which maximum fraction of light is absorbed; $\mathrm{m} / \mathrm{z}=$ mass to charge ratio; $\mathrm{ND}=$ not detected; $\mathrm{D}=$ detected. 


\section{Isolation and comparison of the flavonoid biosynthesis genes in variegated peach flowers}

The coding sequences of six structural genes (CHI, CHS, DFR, F3'H, ANS, and UFGT) from pink and white petals of $P$. persica were obtained using RACE technology (Table S6), and their amino acid sequences are given in Table S7. BLAST results showed that, except for ANS, the coding sequences from both pink and white flower petals share $100 \%$ identity (Table S8), indicating that these genes are homozygous in the peach tree. As for the ANS gene, one 1.071-bp transcript was detected in white flower petals, while two different transcripts, of lengths 1.071 and $942 \mathrm{bp}$, were detected in pink flower petals. The former was $129 \mathrm{bp}$ longer than the latter and the differentially transcribed sequence covered bases 213-341 of the longer transcript (Figure 2).

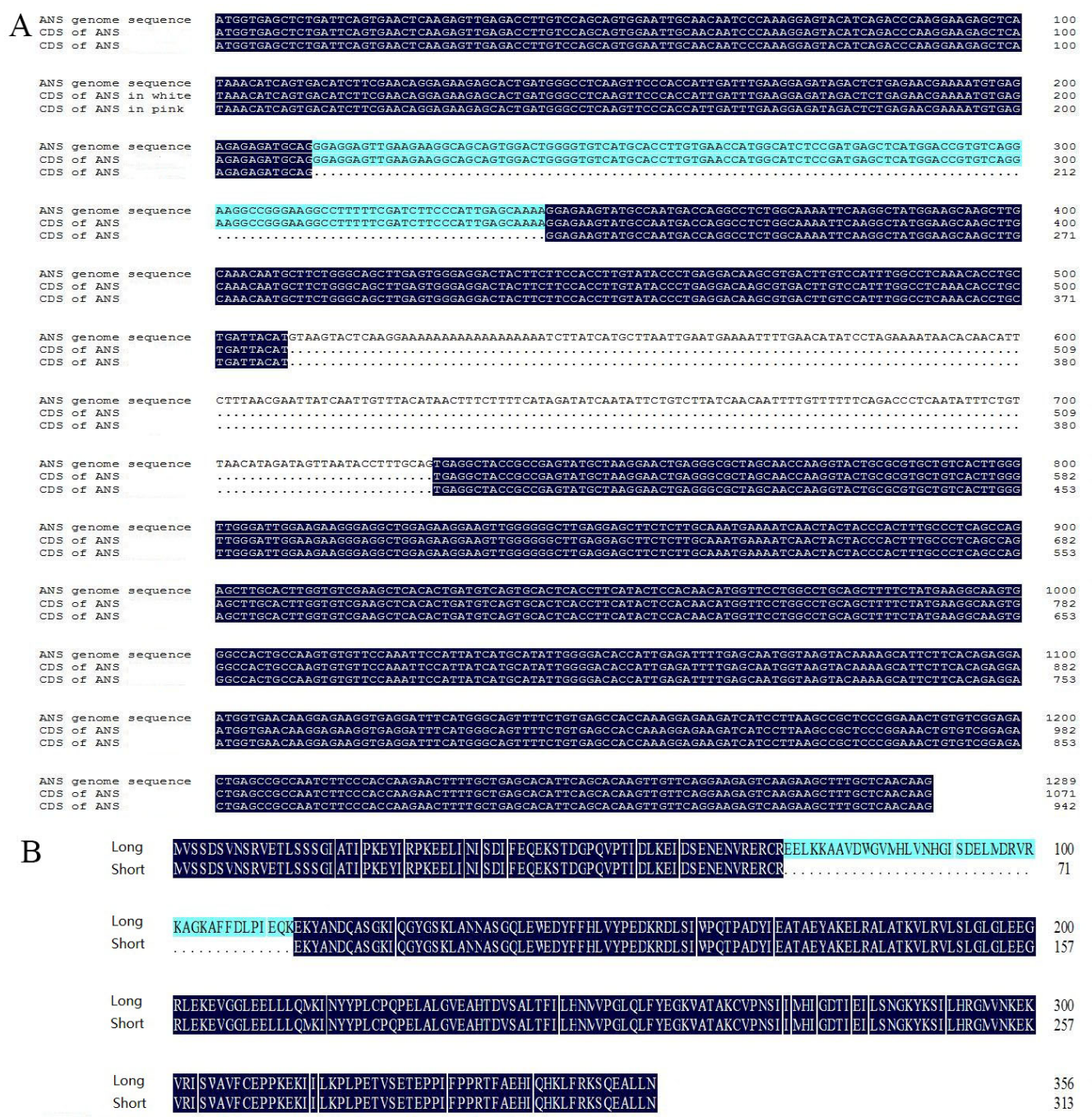

Figure 2. Alignment of long and the short transcripts of the $A N S$ gene. A. Long and short ANS transcripts align with the ANS DNA sequence; B. alignment of the long and short ANS amino acid sequences. 
The longer transcript encoded a 357-amino acid protein and the shorter transcript encoded a 314-amino acid protein. BLASTx analysis against the NCBI protein database showed that the ANS proteins share 99 and $87 \%$ identity, respectively, with the leucoanthocyanidin dioxygenase protein from P. persica (accession No. ABX89941.1) (Table 2). Results of BLASTx analysis for the other five genes are given in Table 2.

Table 2. Putative proteins of flavonoid biosynthetic genes from Prunus persica.

\begin{tabular}{lcclc}
\hline Gene name (accession No.) & Length of the CDS (bp) & Length of the protein (aa) & BLASTx hit (Accession No.) & Identity (\%) \\
\hline CHS (KJ484544; KJ484549) & 1173 & 391 & $\begin{array}{l}\text { Chalcone synthase } \\
\text { (AEJ88217.1) }\end{array}$ & 98 \\
CHI (KJ484543; KJ484548) & 648 & 216 & $\begin{array}{l}\text { Hypothetical protein PRUPE } \\
\text { (EMJ19570.1) }\end{array}$ & 98 \\
F3'H (KJ484546; KJ524274) & 1527 & 509 & $\begin{array}{l}\text { Flavonoid 3' hydroxylase } \\
\text { (AFC62055.1) }\end{array}$ & 99 \\
DFR (KJ484545; KJ484550) & 1038 & 346 & $\begin{array}{l}\text { Dihydroflavonol 4-reductase } \\
\text { (AEJ88220.1) }\end{array}$ & 99 \\
Leucoanthocyanidin dioxygenase & 99 \\
(ABX89941.1) & Leucoanthocyanidin dioxygenase & 87 \\
(ABX89941.1)
\end{tabular}

$\mathrm{bp}=$ base pair; $\mathrm{aa}=$ amino acid.

We further amplified ANS genomic DNA sequences from white and pink petals, which were found to share $100 \%$ identity (Figure S2), indicating that the investigated peach tree is homozygous for the ANS gene. Alignment of the alternative transcripts revealed two introns in the 1.071-bp transcript (Figure 2): the first corresponding to the $129 \mathrm{bp}$ differentially transcribed sequence; the second, spanning a length of 218 bp (bases 510-727 of the genomic sequence). Sequence analysis of the 129-bp intron revealed a GC content of $54.2 \%$. In contrast, GC content was only $24.3 \%$ for the shared intron, which also exhibited the canonical signature GT..AG terminal dinucleotides (Sakabe and de Souza, 2007), a feature absent from the intronic boundary of the 129-bp intron.

Nishihara and Nakatsuka (2011) reported that cis-regulatory elements are likely to play a crucial role in regulating alternative splicing. Thus, we separately amplified 5-kb upstream and downstream sequences flanking the ANS gene from genomic DNA of white and pink flowers, but did not detect any transposon insertion in the regulatory regions of this gene.

\section{Expression of flavonoid biosynthesis genes}

The expression levels of the six structural genes were measured by qRT-PCR. In the flavonoid biosynthetic pathway, synthesis of all flavonoids begins with the precursor, 4-coumaroyl-CoA. Enzymes encoded by the genes $C H S, C H I, F 3^{\prime} H$, and DFR convert this precursor to colorless leucopelargonidin or leucocyanidin (Figure S3). Sequence comparisons between white and pink petals revealed these genes to be completely identical. However, they were all found to be expressed at lower levels in the white petals (Figure 3). Catalyzed by ANS and UFGT, the colorless leucopelargonidin or leucocyanidin are eventually transformed into stable anthocyanin pigments. The $A N S$ gene was observed to be differently spliced in flowers (Figure 2) and its expression was lower in white than in pink petals (Figure 3). 
Similarly for the UFGT gene, although it shared $100 \%$ identity between white and pink petal sequences, its expression was observed to be extremely reduced in the white petals (Figure 3). Since none of the colored pigments were detected in the white petals (Figure 1), we propose that synthesis downstream of leucopelargonidin and leucocyanidin is significantly reduced by the lower expression of the upstream key structural gene (ANS), leading to a lack of substrate for UFGT, and subsequently to the low expression observed in white petals.

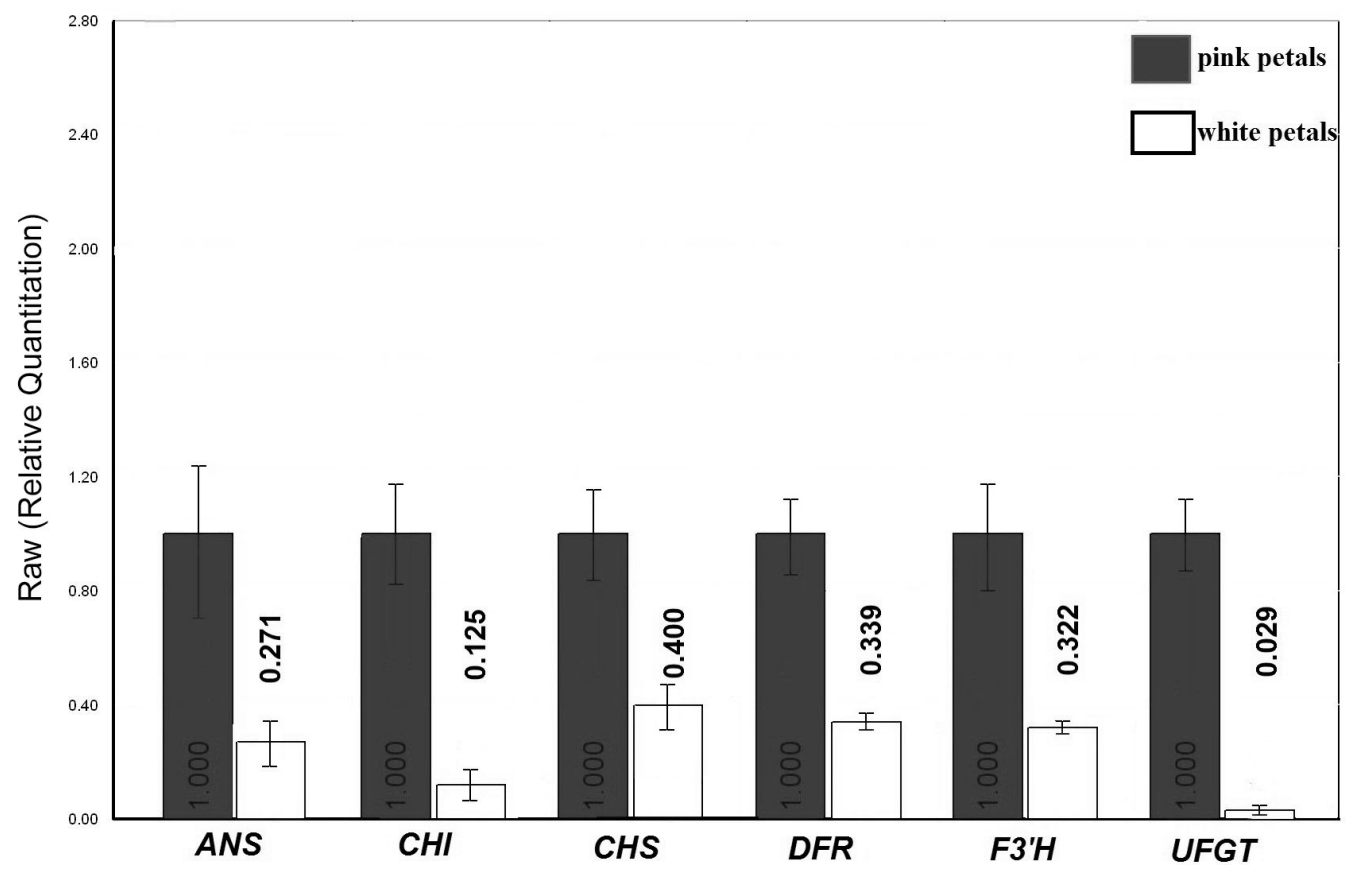

Figure 3. qRT-PCR analysis of the expression levels of the ANS, CHI, CHS, DFR, F3' $H$, and $U F G T$ genes in the white and pink flower petals.

\section{DISCUSSION}

Flavonoids play an important role in the formation and development of flower color (Nakatsuka et al., 2010; Nishihara and Nakatsuka, 2011). The synthesis and accumulation of flavonoids are directly associated with structural and regulatory genes (Nakatsuka et al., 2005). The structural genes involved in this pathway can be classified into two categories: 1) early flavonoid biosynthesis genes, including $\mathrm{CHS}, \mathrm{CHI}$, and $F 3^{\prime} \mathrm{H}$; and 2) late flavonoid biosynthesis genes, such as DFR, ANS, and UFGT (Wei et al., 2011). While peach is heterozygous, these six key structural genes were found to be homozygous in the investigated peach tree; coding sequences revealed no allelic variations among the genes. Sequencing the fulllength $A N S$ gene from genomic DNA revealed no allelic variation, despite the existence of different transcripts. Thus, the different transcripts must have resulted from alternative splicing (AS) instead of allelic differences.

In peach flowers, different variegation patterns are observed: some occur among different flowers on the same branch, while others occur within the same flower (Figure S1). 
Many studies have associated flower color variegation with the insertion of transposable elements into structural genes or regulatory elements related to anthocyanin synthesis. For instance, in Glycine max, a novel exon combination, generated by a 5.7-kb transposon (TgmExpress1) in intron 2 of $\mathrm{F}^{\prime} \mathrm{H}$, causes the plant to produce pink rather than purple flowers (Zabala and Vodkin, 2005). In petunia, a striking variegated flower color mutation arises from the insertion of maize transposable element activator into the Ph6 gene (Chuck et al., 1993). Thus, we examined whether the differentially transcribed 129-bp sequence of the ANS gene represented a kind of short retrotransposable element. Sequence analysis showed that it shared little homology with any known transposable element, and no direct repetitive sequences were found at the boundary of this fragment (Figure 2). Amplification of template DNA also found no allelic variation of the ANS gene at DNA level (Figure S2). Taken together, the results suggest that this fragment was not a transposable insertion.

Since the different $A N S$ transcripts resulted from a homozygous $A N S$ gene, we propose that the 129-bp sequence was retained in the longer ANS transcript as the result of AS. The encoding of multiple proteins by single genes is required to generate the complexity observed in higher eukaryotes (Yura et al., 2006). Genes whose pre-messenger RNAs have multiple alternative splicing locations can produce proteins differing in allosteric regulation, protein localization, and enzymatic activity (Black, 2003). In general, a pre-messenger RNA transcript containing multiple exons can be spliced in different ways. Constitutive exons are always spliced and included in the mature mRNA; exons that are included at times, but excluded at other times, are referred as cassette exons (Black, 2003). In addition, the failure to remove an intron, known as intron retention, is also observed in AS (Black, 2003). Analyses of transcriptional splicing in Arabidopsis thaliana and Oryza sativa indicate that intron retention is a major phenomenon of AS in these species (Ner-Gaon et al., 2007). The retained intron cannot be exon skipping as it is not flanked by introns in the genomic DNA. If a retained intron in the coding regions is in frame with neighboring exons or a stop codon, a shift in the reading frame will result in the synthesis of none functional protein (Sammeth et al., 2008). In this type of AS, a DNA fragment can act either as an intron or as an exon in alternative transcripts of the same gene (Matlin et al., 2005). In humans, retained introns are found to have some unique features, such as high GC content, short sequence length, and weak splice sites (Michael et al., 2005; Sakabe and de Souza, 2007). All these features were observed in the first intron of the $A N S$ gene examined in this study. We propose that the different $A N S$ transcripts detected in pink petals may be due to the intron-retention type of AS.

Alternative splicing allows the $A N S$ gene to be translated into different ANS proteins. ANS protein, which catalyzes the penultimate step in the biosynthesis of anthocyanin, is a 2-oxoglutarate iron-dependent oxygenase enzyme responsible for the synthesis of pelargonidin (color hues from orange to red) and cyanidin (color hues from red to magenta) (Saito et al., 2007; Zhou et al., 2010). The pink color of peach flowers is related to the synthesis of these two pigments. HPLC and LC/MS analyses detected virtually no cyanidin-based or pelargonidin-based anthocyanins in white flower petals. In contrast, high levels of these compounds were detected in pink petals. In the flavonoid biosynthetic pathway, ANS is the key intermediate catalyzing the colorless leucopelargonidin and leucocyanidin to substrates required for the rest of the pathway. However, whether AS of the ANS gene plays a role in flower color variation remains to be confirmed. This question needs to be investigated in different plant species and supported by experimental replication. 
Various transcription factors also regulate the expression levels of flavonoid biosynthesis genes. In a previous study, we detected a number of transcription factors that were differentially expressed in pink and white peach petals (Chen et al., 2014). In this study, we focused on the differential expression of key structural genes. The possibility of differentially expressed transcription factors needs to be explored in a future study. Nevertheless, this study provided essential information regarding the genetic mechanism behind peach flower color variegation.

\section{ACKNOWLEDGMENTS}

Research supported by the Natural Science Foundation of China (\#31270711 and \#31400564), the Jiangsu Natural Science Foundation for Youth (\#BK20130968), the Programs for Innovative Research Team of the Educational Department of China and Universities of Jiangsu Province, and the Priority Academic Program Development (PAPD) of Jiangsu Higher Education Institutions.

\section{Supplementary material}

\section{REFERENCES}

Arnold K, Bordoli L, Kopp J and Schwede T (2006). The SWISS-MODEL workspace: a web-based environment for protein structure homology modeling. Bioinformatics 22: 195-201.

Black DL (2003). Mechanisms of alternative pre-messenger RNA splicing. Ann. Rev. Biochem.. 72: 291-336.

Chaparro JX, Werner D, Whetten R and O'Malley D (1995). Characterization of an unstable anthocyanin phenotype and estimation of somatic mutation rates in peach. J. Hered. 86: 186-193.

Chen Y, Mao Y, Liu H, Yu F, et al. (2014). Transcriptome analysis of differentially expressed genes relevant to variegation in peach flowers. PloS One 9: e90842.

Chuck G, Robbins T, Nijjar C, Ralston ED, at al. (1993). Tagging and cloning of a petunia flower color gene with the maize transposable element activator. Plant Cell 5: 371-378.

De Pascual-Teresa S, Santos-Buelga C and Rivas-Gonzalo JC (2002). LC-MS analysis of anthocyanins from purple corn cob. J. Sci. Food Agr. 82: 1003-1006.

Geourjon C and Deleage G (1995). ANTHEPROT 2.0: a three-dimensional module fully coupled with protein sequence analysis methods. J. Mol. Graphics 13: 209-212.

Guex N and Peitsch MC (1997). SWISS-MODEL and the Swiss-Pdb viewer: an environment for comparative protein modeling. Electrophoresis 18: 2714-2723.

Gutha LR, Casassa LF, Harbertson JF and Naidu RA (2010). Modulation of flavonoid biosynthetic pathway genes and anthocyanins due to virus infection in grapevine (Vitis vinifera L.) leaves. BMC Plant Biol. 10: 187.

Holton TA and Cornish EC (1995). Genetics and biochemistry of anthocyanin biosynthesis. Plant Cell 7: 1071-1083.

Iida S, Hirota T, Morisaki T, Marumoto T, et al. (2004). Tumor suppressor WARTS ensures genomic integrity by regulating both mitotic progression and G1 tetraploidy checkpoint function. Oncogene 23: 5266-5274.

Inagaki Y, Hisatomi Y, Suzuki T, Kasahara K, et al. (1994). Isolation of a Suppressor-mutator/Enhancer-like transposable element, Tpn1, from Japanese morning glory bearing variegated flowers. Plant Cell 6: 375-383.

Jaganath IB and Crozier A (2010). Dietary flavonoids and phenolic compounds. Plant Phenolics Human Health Biochem. Nutr. Pharmacol. 1-49.

Jia N, Shu QY, Wang LS, Dua H, et al. (2008). Analysis of petal anthocyanins to investigate coloration mechanism in herbaceous peony cultivars. Sci. Hortic. 117: 167-173.

Li J, Ou-Lee TM, Raba R, Amundson RG, et al. (1993). Arabidopsis flavonoid mutants are hypersensitive to UV-B irradiation. Plant Cell 5: 171-179.

Liu D, Galli M and Crawford NM (2001). Engineering variegated floral patterns in tobacco plants using the Arabidopsis transposable element Tag1. Plant Cell Physiol. 42: 419-423.

Livak KJ and Schmittgen TD (2001). Analysis of relative gene expression data using real-time quantitative PCR and the 
$2^{-\Delta \Delta C t}$ method. Methods 25: 402-408.

Marchler BA and Bryant SH (2004). Protein domain annotations on the fly. Nucleic Acids Res. 32: 327-331.

Matlin AJ, Clark F and Smith CW (2005). Understanding alternative splicing: towards a cellular code. Nat. Rev. Mol. Cell Biol. 6: 386-398.

Michael IP, Kurlender L, Memari N, Yousef GM, et al. (2005). Intron retention: a common splicing event within the human kallikrein gene family. Clin. Chem. 51: 506-515.

Morata A, Calderón F, González MC, Gómez-Cordovés MC, et al. (2007). Formation of the highly stable pyranoanthocyanins (vitisins A and B) in red wines by the addition of pyruvic acid and acetaldehyde. Food Chem. 100: 1141-1152.

Nakatsuka T, Nishihara M, Mishiba K and Yamamura S (2005). Temporal expression of flavonoid biosynthesis-related genes regulates flower pigmentation in gentian plants. Plant Sci. 168: 1309-1318.

Nakatsuka T, Mishiba K, Kubota A, Abe Y, et al. (2010). Genetic engineering of novel flower color by suppression of anthocyanin modification genes in gentian. Plant Physiol. 167: 231-237.

Ner-Gaon H, Leviatan N, Rubin E and Fluhr R (2007). Comparative cross-species alternative splicing in plants. Plant Physiol. 3: 1632-1641.

Nishihara M and Nakatsuka T (2011). Genetic engineering of flavonoid pigments to modify flower color in floricultural plants. Biotechnol. Lett. 33: 433-441.

Quattrocchio F, Wing J, Leppen HTC, Mol JNM, et al. (1993). Regulatory genes controlling anthocyanin pigmentation are functionally conserved among plant species and have distinct sets of target genes. Plant Cell 5: 1497-1512.

Quattrocchio F, Wing J, van der Woude K, Souer E, et al. (1999). Molecular analysis of the anthocyanin2 gene of petunia and its role in the evolution of flower color. Plant Cell 11: 1433-1444.

Saito K, Kobayashi M, Gong Z, Tanaka Y, et al. (2007). Direct evidence for anthocyanidin synthase as a 2-oxoglutaratedependent oxygenase: molecular cloning and functional expression of cDNA from a red forma of Perilla frutescens. Plant J. 49: 641-654.

Sakabe NJ and de Souza SJ (2007). Sequence features responsible for intron retention in human. BMC Genomics 8: 59.

Sammeth M, Foissac S and Guigó R (2008). A general definition and nomenclature for alternative splicing events. PLoS Comput. Biol. 10: 1371.

Schwede T, Kopp J, Guex N and Peitsch MC (2003). SWISS-MODEL: an automated protein homology-modeling server. Nucleic Acids Res. 31: 3381-3385.

Taylor, LP and Jorgensen R (1992). Conditional male fertility in chalcone synthase-deficient petunia. J. Hered. 83: 11-17. Treutter D (2005). Significance of flavonoids in plant resistance and enhancement of their biosynthesis. Plant Biol. 7 : 581-591.

Verde I, Abbott GA, Scalabrin S, Jung S, et al. (2013). The high-quality draft genome of peach (Prunus persica) identifies unique patterns of genetic diversity, domestication and genome evolution. Nat. Genet. 45: 487-494.

Wei YZ, Hu FC, Hu GB, Li XJ, et al. (2011). Differential expression of anthocyanin biosynthetic genes in relation to anthocyanin accumulation in the pericarp of Litchi chinensis Sonn. PLoS One 6: e19455.

Winkel-Shirley B (2001). Flavonoid biosynthesis: a colorful model for genetics, biochemistry, cell biology and biotechnology. Plant Physiol. 126: 485-493.

Yura K, Shionyu M, Hagino K, Hijikata A, et al. (2006). Alternative splicing in human transcriptome: functional and structural influence on proteins. Gene 2: 63-71.

Zabala G and Vodkin LO (2005). The wp mutation of Glycine max carries a gene-fragment-rich transposon of the CACTA super family. Plant Cell 17: 2619-2632.

Zhou W, Huang C, Gong Y, Feng Q, et al. (2010). Molecular cloning and expression analysis of an ANS gene encoding anthocyanidin synthase from purple-fleshed sweet potato [Ipomoea batatas (L.) Lam]. Plant Mol. Biol. 28: 112-121. 